\title{
Preterm Birth, Maternal Postpartum Depressive Symptoms and Mental and Behavioral Disorders in Children
}

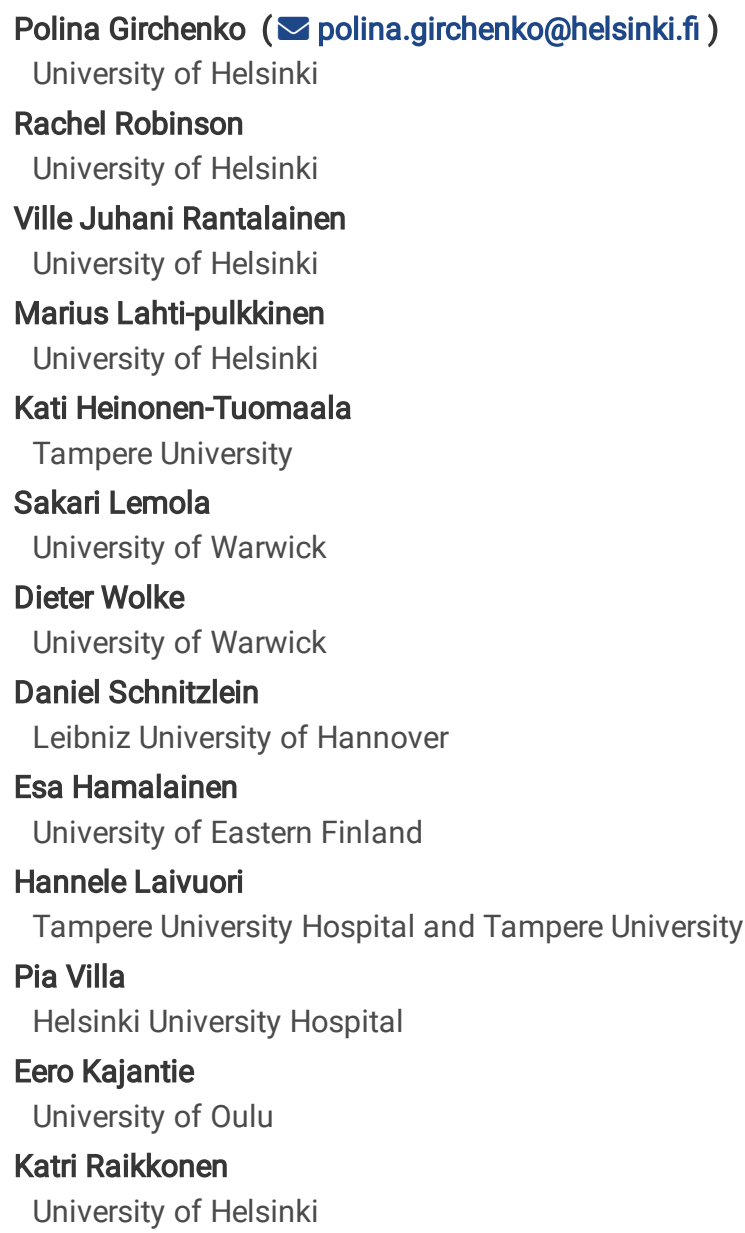

\section{Research Article}

Keywords: postpartum depressive (PPD), PPD symptoms, child mental disorders, depressive symptoms

Posted Date: July 9th, 2021

DOI: https://doi.org/10.21203/rs.3.rs-677953/v1

License: (c) (i) This work is licensed under a Creative Commons Attribution 4.0 International License. Read Full License 


\section{Abstract}

\section{Introduction}

Preterm birth has been linked with postpartum depressive (PPD) disorders and high symptoms levels, but evidence remains conflicting and limited in quality. It remains unclear whether PPD symptoms of mothers with preterm babies were already elevated before childbirth, and whether PPD symptoms mediate/aggravate the effect of preterm birth on child mental disorders. We examined whether preterm birth associated with maternal PPD symptoms, depressive symptoms trajectories from antenatal to postpartum stage, and whether PPD symptoms mediated/aggravated associations between preterm birth and child mental disorders.

\section{Methods}

Mothers of preterm(n=125) and term-born(n=3033) children of the Prediction and Prevention of Preeclampsia and Intrauterine Growth Restriction study reported depressive symptoms four times within 8 weeks before and twice within 12 months after childbirth. Child mental and behavioral disorder diagnoses until age 8.4-12.8 years came from medical register.

\section{Results}

Preterm birth associated with higher PPD symptoms $(M D=0.19 \mathrm{SD}, 95 \% \mathrm{Cl} 0.01,0.37, \mathrm{p}=0.04)$, and higher odds $(\mathrm{OR}=2.23,95 \% \mathrm{Cl}$ $1.22,4.09, p=0.009$ ) of the mother to belong to a group that had consistently high depressive symptoms levels trajectory from antenatal to postpartum stage. PPD symptoms partially mediated and aggravated the association between preterm birth and child mental disorders.

\section{Conclusions}

Preterm birth, maternal PPD symptoms and child mental disorders are associated, calling for timely prevention interventions.

\section{Introduction}

Preterm birth (<37 completed weeks of gestation) is the leading cause of perinatal morbidity and mortality ${ }^{1}$. It poses a risk for neurosensory impairments, and for physical, mental and behavioural disorders later in life ${ }^{2-5}$. Preterm birth also induces substantial challenges and distress among the parents of the preterm infants ${ }^{6}$. Hence, preterm birth has been implicated as a risk factor for maternal postpartum depression (PPD) ${ }^{7}$.

However, high-quality studies on the association between preterm birth and maternal PPD have been scarce. Using the Newcastle-Ottawa Scale (NOS) ${ }^{8}$, we systematically assessed the quality of evidence of previous studies testing the association between preterm birth and maternal PPD (Tables ST1 and ST2). The NOS assessment revealed the limited quality of the available evidence: of the 26 reviewed cohort and cross-sectional studies, 16 (61.5\%) were found of low quality, five (19.2\%) of moderate and five (19.2\%) of high quality (ST1, ST2). Of the five high-quality studies, three were population-based register studies with focus on maternal PPD disorder diagnoses up to 12 months after childbirth ${ }^{9-11}$. Two of the register studies ${ }^{10,11}$ found an association between preterm birth and maternal PPD, and one ${ }^{9}$ reported a null association. The other two high-quality studies were cohort studies with focus on maternal PPD symptoms. One of the cohort studies did not find an association between preterm birth and maternal PPD symptoms two months after childbirth ${ }^{12}$, while the other did, but used one nonvalidated question addressing maternal low mood, depression and hopelessness $2-4$ months after childbirth as an indicator of PPD ${ }^{13}$. This calls for further high-quality studies on preterm birth and PPD symptoms measured using validated scales. Furthermore, as depressive symptoms show high continuity from the antenatal to the postpartum stage (Lahti et al-2017), further studies are also needed that examine if PPD symptoms of mothers with preterm babies were already elevated in the antenatal stage.

Apart from the implicated association with preterm birth, maternal PPD has been also linked with the child's higher risk for mental and behavioral disorders ${ }^{14,15}$, which suggests that PPD may be on a pathway between preterm birth and mental and behavioral disorders in children. One previous study has reported that maternal mood disorder diagnoses after childbirth mediated the association between lower gestational age and child risk for anxiety disorders at preschool age ${ }^{16}$. However, we are not aware of studies that have tested whether maternal PPD symptoms mediate the associations between preterm birth and child mental and behavioral disorders. It also remains unknown if maternal PPD aggravates the association of preterm birth with these child mental health outcomes.

We examined in a large pregnancy cohort of Finnish women and their children if preterm birth was associated with maternal PPD symptoms and probable clinical PPD from the childbirth until 12 months postpartum. Further, to unravel if the depressive symptoms levels of women with a preterm baby were high already in the antenatal stage, we studied if preterm birth was associated with depressive symptoms trajectories from the antenatal and the postpartum stage. We also examined if maternal PPD symptoms mediated the association between 
preterm birth and mental and behavioral disorders in the children until mid-childhood. Finally, we examined if the effects of preterm birth and maternal PPD on mental and behavioral disorders in the children were additive.

\section{Methods Participants}

The Prediction and Prevention of Preeclampsia and Intrauterine Growth Restriction (PREDO) study is described in detail elsewhere 22 . In brief, it comprises 4777 mothers who gave birth to a singleton live-born child in Finland between 2006-2010. Recruitment took place at ten study hospitals in Southern/Eastern Finland at the first fetal ultrasound screening in early pregnancy. Three women have since withdrawn from the study. We excluded 6 mother-child dyads with unknown gestational age, 29 with missing Finnish Medical Birth Register (MBR) data, 178 with post-term birth (at gestational age $\geq 42^{+0}$ weeks), and 4 whom we did not have data on child mental or behavioral disorders from the Finnish Care Register for Health Care (HILMO). Of the 4559 women, 3158 (69.3\%) completed questionnaires on depressive symptoms twice up to 12 months postpartum, and of them 125 (4.0\%) gave preterm birth.

Diagnoses of any mental disorder from birth until 31st December 2018, when the children were 8.4-12.8 (median 10.2, interquartile range (IRQ) 9.5-10.8) years of age, were available for all children of the 3158 women.

Characteristics of the women who did provide the data on PPD and those who did not are shown in the table ST3. All participating women signed an informed consent. The consent enabled linkage to nationwide medical register data for the women and children using unique personal identification numbers assigned to all Finnish citizens and residents since 1971. The PREDO study protocol was approved by the Ethics Committees of the Helsinki and Uusimaa Hospital District and recruitment was conducted with permission from the participating study hospitals. All participants provided written informed consent. Consent of participating children was provided by parent(s)/legal guardian(s).

\section{Measures}

Preterm birth

Gestational age was derived from hospital records and/or MBR. We defined preterm birth as birth at $36^{+6}$ gestational weeks + days or less and term birth as birth between $37^{+0}$ and $41^{+6}$ gestational weeks + days.

Maternal PPD and antenatal depressive symptoms

The women completed the Center for Epidemiologic Studies Depression Scale (CES-D) ${ }^{23}$ twice up to 12 months postpartum (time 1 : median 2.1 weeks, IRQ 2.0-2.4 weeks postpartum; time 2: 6.4 months, IRQ 6.1-7.3 months postpartum). The 20 CES-D questions were rated on a scale from none ( 0 ) to all of the time (3). Higher scores indicate more depressive symptoms during the past week. We defined PPD symptoms as the mean of the two postpartum CES-D scores and probable clinical PPD as mean CES-D $\geq 16$.

Using the CES-D, the women also rated prospectively biweekly their antenatal depressive symptoms up to 14 times starting from $12-14$ gestational week until delivery for women who gave birth to a preterm baby and until 38-39 gestational weeks for women who gave birth to a full-term baby. Of these biweekly CES-D measurements we identified the four consecutive measurements before childbirth: we have previously shown in this sample that in women who gave birth to a preterm baby, depressive symptoms begin increasing approximately 8 weeks before childbirth ${ }^{24}$, a timeframe which is captured by the four biweekly consecutive CES-D measurement points before childbirth.

Mental and behavioral disorders in children

We identified diagnoses of mental and behavioral disorders from the HILMO from the birth of the child between 11/07/2006 and 07/24/2010 to $12 / 31 / 2018$. The HILMO includes primary and subsidiary diagnoses of all inpatient hospital treatments and outpatient visits in specialized medical care coded using the International Statistical Classification of Diseases and Related Health Problems-10 (ICD-10). HILMO is a valid tool for research ${ }^{25}$. We studied any mental and behavioral disorder (ICD-10: F00-F99) as the outcome.

\section{Covariates}

The following variables were included as covariates: maternal age at childbirth (years), smoking at any time during pregnancy (no/any smoking during pregnancy), mode of delivery (vaginal delivery/caesarean section), child's sex (male/female) and age (years) at first mental and behavioral disorder diagnosis with data extracted from hospital records, MBR and/or HILMO; maternal education level (secondary or less (International Standard Classification of Education (ISCED) levels 0 to 5)/tertiary (ISCED levels 6 to 8 ) and alcohol use (no/any alcohol use during pregnancy) were self-reported in early pregnancy. Smoking and alcohol use were combined into one variable representing smoking

Page $3 / 11$ 
and/or alcohol use at any time during pregnancy (yes/no). Maternal mood disorder diagnoses before the childbirth were identified from HILMO (ICD-10 codes F32-F33, F341 since 1996 and ICD-9 codes 2961, 2968A, and 3004A between 1987-1995).

\section{Statistical analyses}

Associations between preterm birth and maternal PPD symptoms levels were tested using linear regression and with probable clinical PPD using logistic regression.

To identify trajectories of maternal depressive symptoms between antenatal and postpartum stage, we conducted latent class analysis (LCA) based on their four antenatal and two postpartum CES-D scores. We compared solutions with two to six latent classes. Based on criteria for the optimal number of classes described by Kongsted and Nielsen ${ }^{26}$, the optimal solution was based on (1) goodness-of-fit criteria (Akaike Information Criterion [AIC], Bayesian Information Criterion [BIC]), (2) reasonable distribution of participants across subgroups (at least 10\% of the sample), (3) high certainty of classification identified by posterior probabilities, and (4) clear clinical characteristics of the participants within each of the subgroups. Using the optimal LCA solution, we then tested whether the women who gave birth to a preterm and term baby differed in their odds of belonging to groups differing in their depressive symptoms trajectories identified by the LCA.

To study whether maternal PPD symptoms mediated the association between preterm birth and mental and behavioral disorders in the children, we used SPSS PROCESS macro with 5000 bootstrapped samples ${ }^{27}$. Before testing mediation, we ensured that the criteria for mediation were met, namely that the predictor, mediator and outcome variables were associated. Therefore, before proceeding to mediation analyses we also examined if preterm birth and maternal PPD symptoms were associated with mental and behavioral disorders in children by using Cox proportional hazards models. The proportionality assumptions were met (tests for the proportional hazards assumption yielded pvalues $\geq 0.07$ ).

We then examined if preterm birth and maternal PPD symptoms had additive effects on the hazard of mental and behavioral disorders in children by applying Cox proportional hazards models. For this analysis, we compared the hazards of mental and behavioral disorders in the children who were born at term and whose mothers reported no probable clinical PPD (referent) with (a) children born at term and whose mothers reported probable clinical PPD, (b) preterm children whose mothers reported no probable clinical PPD and (c) preterm children whose mothers reported probable clinical PPD.

We report the associations as unadjusted and adjusted for all covariates. Additionally, in examining the associations between preterm birth and PPD, we conducted sensitivity analyses in which we excluded women with a previous history of mood disorders diagnosed before childbirth. Statistical analyses were conducted by using SAS 9.4 (SAS Institute, Inc., Cary, NC, USA), Stata 15 (StataCorp. 2017. Stata Statistical Software: Release 15. College Station, TX: StataCorp LLC) and SPSS-IBM (Software, v.24.0 SPSS).

\section{Results}

Descriptive characteristics of the preterm and term born children and their mothers are shown in Table 1. Of the preterm born children $88 \%$ (n $=110)$ were born moderately or late preterm $\left(32^{+0}-36^{+6}\right.$ gestational weeks) and $12 \%(n=15)$ were born very or extremely preterm $\left(<32^{+} 0\right.$ gestational weeks). Preterm children were more often delivered via Caesarean section, they were more often boys, and their mothers reported higher levels of antenatal depressive symptoms 8 weeks before childbirth (Table 1). Associations between the covariates and maternal PPD symptoms and mental and behavioral disorders in children are presented in Table ST4. 


\begin{tabular}{|c|c|c|c|}
\hline & \multicolumn{3}{|c|}{ Mean (SD) or N (\%) } \\
\hline & $\begin{array}{l}\text { Term birth } \\
(N=3033)\end{array}$ & $\begin{array}{l}\text { Preterm birth } \\
(\mathrm{N}=125)\end{array}$ & $\mathbf{P}$ \\
\hline \multicolumn{4}{|l|}{ Maternal characteristics } \\
\hline Maternal age at delivery, years & $31.8(4.7)$ & $31.7(4.8)$ & 0.87 \\
\hline Data not available & 0 & 0 & \\
\hline Education level & & & 0.81 \\
\hline Upper secondary or less & $1226(40.5 \%)$ & $52(41.6 \%)$ & \\
\hline Tertiary & $1801(59.5 \%)$ & $73(58.4 \%)$ & \\
\hline Data not available & $6(0.2 \%)$ & 0 & \\
\hline Smoking or alcohol use at any point during pregnancy & & & 0.81 \\
\hline No & $2347(77.4 \%)$ & $99(79.2 \%)$ & \\
\hline Yes & $648(22.6 \%)$ & $24(20.8 \%)$ & \\
\hline Data not available & $38(1.3 \%)$ & $2(1.6 \%)$ & \\
\hline Mode of delivery & & & $<0.0001$ \\
\hline Vaginal & $2532(83.8 \%)$ & $76(61.3 \%)$ & \\
\hline Caesarean section & $490(16.2 \%)$ & $48(38.7 \%)$ & \\
\hline Data not available & $11(0.4 \%)$ & $1(0.8 \%)$ & \\
\hline Antenatal depressive symptoms 6-8 weeks before delivery (continuous CES-D score) & $11.8(7.2)$ & $13.3(8.4)$ & 0.02 \\
\hline Data not available & $130(4.3 \%)$ & $5(4.0 \%)$ & \\
\hline Antenatal probable clinical depression $6-8$ weeks before delivery (CES-D $\geq 16$ ) & & & 0.04 \\
\hline No & $2182(75.2 \%)$ & $80(66.7 \%)$ & \\
\hline Yes & $721(24.8 \%)$ & $40(32.3 \%)$ & \\
\hline Data not available & $1407(32.2 \%)$ & $65(34.4 \%)$ & \\
\hline $\begin{array}{l}\text { Postpartum depressive symptoms up to } 12 \text { months after childbirth (continuous CES-D } \\
\text { score) }\end{array}$ & $10.2(7.0)$ & $11.7(7.2)$ & 0.02 \\
\hline Data not available & 0 & 0 & \\
\hline Postpartum probable clinical depression up to 12 months after childbirth (CES-D $\geq 16$ ) & & & 0.34 \\
\hline No & $2458(81.0 \%)$ & $97(77.6 \%)$ & \\
\hline Yes & $575(19.0 \%)$ & $28(22.4 \%)$ & \\
\hline Data not available & 0 & 0 & \\
\hline Mood disorder diagnoses before childbirth & & & 0.88 \\
\hline No & $2896(95.5 \%)$ & $119(95.2 \%)$ & \\
\hline Yes & $137(4.5 \%)$ & $6(4.8 \%)$ & \\
\hline Data not available & 0 & 0 & \\
\hline \multicolumn{4}{|l|}{ Child characteristics } \\
\hline \multicolumn{4}{|l|}{ Child sex } \\
\hline Boy & $1552(51.2 \%)$ & $77(61.6 \%)$ & 0.02 \\
\hline
\end{tabular}




\begin{tabular}{|c|c|c|c|}
\hline & Mean (SD) or I & & \\
\hline & $\begin{array}{l}\text { Term birth } \\
(\mathrm{N}=3033)\end{array}$ & $\begin{array}{l}\text { Preterm birth } \\
(\mathrm{N}=125)\end{array}$ & $\mathbf{P}$ \\
\hline Girl & $1481(48.8 \%)$ & $48(38.4 \%)$ & \\
\hline Data not available & 0 & 0 & \\
\hline Gestational age at birth, weeks & $40.0(1.1)$ & $35.0(2.1)$ & $<0.0001$ \\
\hline Data not available & 0 & 0 & \\
\hline Follow-up length years (Median, IRQ) & $\begin{array}{l}9.7(9.5- \\
10.7)\end{array}$ & $\begin{array}{l}9.7(9.6- \\
10.9)\end{array}$ & 0.65 \\
\hline Data not available & 0 & 0 & \\
\hline Mental and behavioral disorder diagnosis & & & 0.02 \\
\hline No & $2720(89.7 \%)$ & $104(82.2 \%)$ & \\
\hline Yes & $313(10.3 \%)$ & $21(16.8 \%)$ & \\
\hline Data not available & 0 & 0 & \\
\hline
\end{tabular}

\section{Preterm birth and maternal PPD symptoms}

In unadjusted and adjusted models, preterm birth was associated with higher levels of maternal PPD symptoms, but not with higher odds of maternal probable clinical PPD (Table 2). When we excluded women with mood disorders diagnosed before childbirth from the analyses, the associations remained significant (Table 2).

Table 2

Associations between preterm versus term birth and maternal postpartum depressive symptoms (PPD)

\begin{tabular}{|c|c|c|c|c|c|c|c|c|c|c|c|c|}
\hline \multirow{3}{*}{$\begin{array}{l}\text { Preterm } \\
\text { versus } \\
\text { term } \\
\text { birth }\end{array}$} & \multirow{2}{*}{\multicolumn{3}{|c|}{ Level of PPD symptoms }} & \multirow{2}{*}{\multicolumn{3}{|c|}{ Probable clinical PPD }} & \multicolumn{6}{|c|}{$\begin{array}{l}\text { Latent classes of women based on antenatal and PPD } \\
\text { symptoms }\end{array}$} \\
\hline & & & & & & & \multicolumn{3}{|c|}{$\begin{array}{l}\text { Consistently moderate } \\
\text { versus low }\end{array}$} & \multicolumn{3}{|c|}{ Consistently high versus low } \\
\hline & $\begin{array}{l}\text { Mean } \\
\text { difference } \\
\text { (SD units) }\end{array}$ & $\begin{array}{l}95 \% \\
\text { Confidence } \\
\text { Interval }\end{array}$ & $\mathbf{P}$ & $\begin{array}{l}\text { Odds } \\
\text { Ratio }\end{array}$ & $\begin{array}{l}95 \% \\
\text { Confidence } \\
\text { Interval }\end{array}$ & $\mathbf{P}$ & $\begin{array}{l}\text { Odds } \\
\text { Ratio }\end{array}$ & $\begin{array}{l}95 \% \\
\text { Confidence } \\
\text { Interval }\end{array}$ & $\mathbf{P}$ & $\begin{array}{l}\text { Odds } \\
\text { Ratio }\end{array}$ & $\begin{array}{l}95 \% \\
\text { Confidence } \\
\text { Interval }\end{array}$ & $\mathbf{P}$ \\
\hline $\begin{array}{l}\text { Model } \\
1\end{array}$ & 0.22 & $0.04,0.40$ & 0.02 & 1.23 & $0.80,1.90$ & 0.34 & 1.70 & $0.96,2.99$ & 0.07 & 2.25 & $1.24,4.10$ & 0.008 \\
\hline $\begin{array}{l}\text { Model } \\
2\end{array}$ & 0.19 & $0.01,0.37$ & 0.04 & 1.14 & $0.74,1.77$ & 0.55 & 1.69 & $0.95,3.00$ & 0.07 & 2.23 & $1.22,4.09$ & 0.009 \\
\hline $\begin{array}{l}\text { Model } \\
3\end{array}$ & 0.19 & $0.01,0.38$ & 0.04 & 1.16 & $0.74,1.83$ & 0.52 & 1.65 & $0.93,2.93$ & 0.09 & 2.25 & $1.22,4.15$ & 0.009 \\
\hline \multicolumn{13}{|c|}{$\begin{array}{l}\text { Model } 1 \text { is unadjusted; Model } 2 \text { is adjusted for maternal age at delivery, maternal education, maternal smoking and/or alcohol use at any } \\
\text { time during pregnancy, delivery mode and child sex; Model } 3 \text { is a sensitivity analyses: Model } 2 \text { where women with mood disorder } \\
\text { diagnoses before childbirth are excluded }\end{array}$} \\
\hline
\end{tabular}

The optimal LCA solution identified three groups of women who differed in depressive symptoms levels trajectories between the antenatal and postpartum stage (Table ST5). In all three groups, levels of depressive symptoms showed high stability: the levels were consistently low ( $\mathrm{n}=$ $633,20.0 \%)$, moderate $(n=1696,53.7 \%)$ or high $(n=829,26.3 \%)$ between the antenatal and postpartum stage (Figure S1). Women in the group with consistently high levels of depressive symptoms between the antenatal and postpartum stage had depressive symptoms scores that were consistently at or above the probable clinical cutoff (CES-D $\geq 16)$ (Figure S1). 
In unadjusted and adjusted models, preterm birth was associated with significantly higher odds of the mother to belong to the group who had a consistently high versus low symptoms levels trajectory between the antenatal and postpartum stage (Table 2). The corresponding odds of the mother to belong to the group who had a consistently moderate versus low symptoms levels trajectory between the antenatal and postpartum stage was not statistically significant (Table 2).

Maternal PPD symptoms as a mediator between preterm birth and mental and behavioral disorders in children

Table 3 shows that both preterm birth and higher levels of maternal PPD symptoms were associated with significantly higher hazards of mental and behavioral disorders in children. Mediation analyses indicated that maternal PPD symptoms partially mediated the association between preterm birth and mental and behavioral disorders in children (Fig. 1) with the effect size proportion mediated being $6.7 \%$ in unadjusted and $7.8 \%$ in adjusted models.

Table 3

Associations between preterm birth and maternal postpartum depressive symptoms (PPD) with mental and behavioral disorders in children in a follow-up to 8.4-12.8 years of age.

\begin{tabular}{|c|c|c|c|}
\hline & \multicolumn{3}{|c|}{ Mental and behavioral disorders in children } \\
\hline & Hazard Ratio & 95\% Confidence Interval & $\mathbf{P}$ \\
\hline \multicolumn{4}{|c|}{ Preterm versus term birth } \\
\hline Model 1 & 1.61 & $1.04,2.51$ & 0.03 \\
\hline Model 2 & 1.57 & $1.00,2.45$ & 0.05 \\
\hline \multicolumn{4}{|c|}{ Maternal PPD symptoms (continuous CES-D score) (SD units) } \\
\hline Model 1 & 1.26 & $1.14,1.41$ & $<0.0001$ \\
\hline Model 2 & 1.24 & $1.12,1.38$ & $<0.0001$ \\
\hline \multicolumn{4}{|c|}{ Maternal probable clinical PPD (CES-D $\geq 16$ versus CES-D < 16) } \\
\hline Model 1 & 1.51 & $1.18,1.93$ & 0.0009 \\
\hline Model 2 & 1.47 & $1.15,1.88$ & 0.002 \\
\hline
\end{tabular}

Additive effects of preterm birth and maternal PPD symptoms on mental and behavioral disorders in children

Figure 2 shows that compared to term-born children born whose mothers had no probable clinical PPD, the hazard of mental and behavioral disorders was $46 \%$ higher for children born preterm and whose mother reported no probable clinical PPD, 45\% higher for children born at term and whose mothers reported probable clinical PPD, and $161 \%$ higher for children born preterm and whose mothers reported probable clinical PPD. The effects of preterm birth and maternal PPD were additive, as the child's hazard for mental and behavioral disorders increased linearly according to the number of these exposures ( $0=$ term, no PPD, $1=$ term and PPD or preterm and no PPD, $2=$ preterm and PPD) (Fig. 2$)$.

\section{Discussion}

This study showed that mothers whose babies were born preterm had higher levels of PPD symptoms up to 12 months postpartum. Preterm birth was not associated with maternal probable clinical PPD, pointing to only subtle differences in the levels of depressive symptoms between the mothers who gave birth to preterm and term babies. However, when we took into account the continuity of maternal depressive symptoms from the antenatal to the postpartum periods, mothers who gave birth to preterm babies had over 2-fold higher odds to belong to the group which displayed consistently high depressive symptoms, which were at or above the probable clinical cutoff between the antenatal and the postpartum stage. These findings suggest that even though the differences appear to be subtle in the levels of PPD symptoms between mothers who gave birth to preterm and term babies, these differences are aggravated by high levels of antenatal depressive symptomatology persisting to the postpartum stage.

These findings are consistent with the one high-quality cohort study from the US, which found that preterm birth was associated with maternal feelings of low mood, depression and hopelessness $2-4$ months after childbirth ${ }^{13}$. They are also in partial agreement with the other highquality cohort study from Greece, which found no associations between preterm birth and maternal probable clinical PPD measured two 
months after childbirth with the EPDS ${ }^{12}$. Neither of these studies followed the women longer than 2-4 months after childbirth, nor studied trajectories of depressive symptoms from the antenatal to the postpartum stage, as we did, therefore, our study adds to high-quality evidence.

Our findings indicate that maternal PPD symptoms partially mediate the association between preterm birth and the risk of mental and behavioral disorders in children. The effect size proportion mediated is, however, less than $8 \%$, suggesting that maternal PPD symptomatology is only a modest mediator of this association. At the same time, both preterm birth, maternal PPD symptoms and probable clinical PPD are associated with the child's higher hazard of mental and behavioral disorders, and their effects appear to be additive. While we and others have also shown that preterm birth ${ }^{2-5}$ and maternal PPD ${ }^{14,17}$ were associated with higher mental health risks in children, we are not aware of previous reports showing that these risks were additive.

Our results suggest that while it is difficult to prevent preterm birth in itself ${ }^{18}$, it would be feasible to target maternal PPD symptoms. The continuity of depressive symptoms from the antenatal to the postpartum stage, however, highlights that preventive interventions that focus on maternal depressive symptomatology should start already in pregnancy. Further benefits may be gained by postpartum interventions, as it has been shown that intervention programs that focus on decreasing stress in the parents of preterm infants also reduce the risk of maternal PPD (Swartz, Frank et al. 2008, Wickramaratne, Gameroff et al. 2011). Addressing PPD in women who gave birth to preterm babies should alleviate the adverse effects of preterm birth: even a small reduction of the adverse effect of preterm birth should result in considerable public health benefits on a population level. Furthermore, reduction of maternal PPD symptoms in women who gave birth to preterm babies should lessen additive effects of preterm birth and PPD on children' mental health risks.

The ability to verify that the associations between preterm birth and maternal PPD symptoms were not explained by maternal history of mood disorders and the ability to take into account the continuity of depressive symptomatology from the antenatal to the postpartum stage, both strong predictors of maternal PPD 10,14,19, are key strengths in this study. Other study strengths include prospective study design, large and well-characterized sample, longitudinal measurement of depressive symptoms from the antenatal to the postpartum stage, which allowed us to identify maternal antenatal depressive symptoms before preterm and term delivery, data on maternal mood and child mental and behavioral disorder diagnoses extracted from nationwide registers and null data attrition in child follow-up.

However, our study does not unravel the mechanisms underlying the association between preterm birth and maternal PPD symptoms, and mechanisms underlying their association with increased risk of mental and behavioral disorders in children. While data attrition in the child follow-up was null, our study suffers from selective dropout, as the women who participated in the postpartum follow-up and reported PPD symptoms were older, used substances less, and less often had been diagnosed with mood disorders than the women who did not. However, even selective subject loss has been found to only marginally alter predictive models ${ }^{20}$. Nevertheless, it may limit generalizability of our findings to less advantageous samples. A further limitation to generalizability is the high-resource Nordic setting. Finally, over $80 \%$ of the preterm births in our study were moderate or late preterm, which is in alignment with the population prevalence of moderate and late preterm births in Finland ${ }^{21}$. It is, hence, possible, that the associations might appear different in very and extremely preterm groups.

In conclusion, our findings suggest that preterm birth and maternal PPD symptoms are associated. The continuity of depressive symptomatology from antenatal to postpartum period and its associations with preterm birth suggests that addressing depressive symptoms already in pregnancy may have beneficial effects on the risk of preterm birth, PPD and subsequently on child mental and behavioral disorders. Mediation and additive effects of preterm birth and maternal PPD on child's higher risk for mental and behavioral disorders call for prevention interventions aimed at reducing PPD symptoms in women who gave preterm birth.

\section{Declarations}

\section{Authors contributions}

1. Conception and design, or acquisition of data, or analysis and interpretation of data (Drs. Girchenko, Rantalainen, Lahti-Pulkkinen, Heinonen-Tomaala Hämäläinen, Villa, Kajantie, Laivuori, Räikkönen and Ms. Robinson);

2. Drafting the article or revising it critically for important intellectual content (Drs. Girchenko, Rantalainen, Lahti-Pulkkinen, Heinonen-Tomaala, Lemola, Wolke, Schnitzlein, Hämäläinen, Villa, Kajantie, Laivuori, Räikkönen and Ms. Robinson);

3. Final approval of the version to be published (Drs. Girchenko, Rantalainen, Lahti-Pulkkinen, Heinonen-Tomaala, Lemola, Wolke, Schnitzlein, Hämäläinen, Villa, Kajantie, Laivuori, Räikkönen and Ms. Robinson).

\section{Acknowledgements}


The PREDO study would not have been possible without the dedicated contribution of the PREDO Study group members: A Aitokallio-Tallberg, A-M Henry, VK Hiilesmaa, T Karipohja, R Meri, S Sainio, T Saisto, S Suomalainen-Konig, V-M Ulander, T Vaitilo (Department of Obstetrics and Gynaecology, University of Helsinki and Helsinki University Central Hospital, Helsinki, Finland), L Keski-Nisula, Maija-Riitta Orden (Kuopio University Hospital, Kuopio Finland), E Koistinen, T Walle, R Solja (Northern Karelia Central Hospital, Joensuu, Finland), M Kurkinen (PäijätHäme Central Hospital, Lahti, Finland), P.Taipale. P Staven (lisalmi Hospital, lisalmi, Finland), J Uotila (Tampere University Hospital, Tampere, Finland). We also thank the PREDO cohort mothers, fathers and children for their enthusiastic participation.

\section{Additional information}

\section{Competing interests statement}

The authors have no competing interests to disclose.

\section{Ethical standards}

The authors assert that all procedures contributing to this work comply with the ethical standards of the relevant national and institutional committees on human experimentation and with the Helsinki Declaration of 1975, as revised in 2008.

\section{References}

1. Chawanpaiboon, S. et al. Global, regional, and national estimates of levels of preterm birth in 2014: a systematic review and modelling analysis. Lancet Glob Health, 7, e37-e46 https://doi.org/10.1016/s2214-109x(18)30451-0 (2019).

2. Ream, M. A. \& Lehwald, L. Neurologic Consequences of Preterm Birth. Current Neurology and Neuroscience Reports, 18,48 https://doi.org/10.1007/s11910-018-0862-2 (2018).

3. Pyhälä, R. et al. Self-Reported Mental Health Problems Among Adults Born Preterm: A Meta-analysis., 139, e20162690 https://doi.org/10.1542/peds.2016-2690 (2017).

4. Moster, D., Lie, R. T. \& Markestad, T. Long-Term Medical and Social Consequences of Preterm Birth. New England Journal of Medicine, 359, 262-273 https://doi.org/10.1056/NEJMoa0706475 (2008).

5. Bachmann, C. S., Risnes, K., Bjørngaard, J. H., Schei, J. \& Pape, K. Association of Preterm Birth With Prescription of Psychotropic Drugs in Adolescence and Young Adulthood. JAMA Network Open, 4, e211420-e211420 https://doi.org/10.1001/jamanetworkopen.2021.1420 (2021).

6. Yaari, M., Treyvaud, K., Lee, K. J., Doyle, L. W. \& Anderson, P. J. Preterm Birth and Maternal Mental Health: Longitudinal Trajectories and Predictors. Journal of Pediatric Psychology, 44, 736-747 https://doi.org/10.1093/jpepsy/jsz019 (2019).

7. de Paula Eduardo, J. A. F., de Rezende, M. G., Menezes, P. R. \& Del-Ben, C. M. Preterm birth as a risk factor for postpartum depression: A systematic review and meta-analysis. Journal of Affective Disorders, 259, 392-403 https://doi.org/10.1016/j.jad.2019.08.069 (2019).

8. Stang, A. J. E. J. o. E. Critical evaluation of the Newcastle-Ottawa scale for the assessment of the quality of nonrandomized studies in meta-analyses. 25,603-605, doi:10.1007/s10654-010-9491-z (2010).

9. Meltzer-Brody, S. et al. Obstetrical, pregnancy and socio-economic predictors for new-onset severe postpartum psychiatric disorders in primiparous women. Psychol Med, 47, 1427-1441 https://doi.org/10.1017/s0033291716003020 (2017).

10. Silverman, M. E. et al. The risk factors for postpartum depression: A population-based study. Depress Anxiety, 34, 178-187 https://doi.org/10.1002/da.22597 (2017).

11. Youn, H. et al. Obstetric risk factors for depression during the postpartum period in South Korea: a nationwide study. $J$ Psychosom Res, 102, 15-20 https://doi.org/10.1016/j.jpsychores.2017.09.003 (2017).

12. Koutra, K. et al. Pregnancy, perinatal and postpartum complications as determinants of postpartum depression: the Rhea mother-child cohort in Crete, Greece. Epidemiol Psychiatr Sci, 27, 244-255 https://doi.org/10.1017/s2045796016001062 (2018).

13. Barber, K. S. et al. The Association between Having a Preterm Birth and Later Maternal Mental Health: An Analysis of U.S. Pregnancy Risk Assessment Monitoring System Data. Women's Health Issues, https://doi.org/10.1016/j.whi.2020.08.007 (2020).

14. Lahti, M. et al. Maternal Depressive Symptoms During and After Pregnancy and Psychiatric Problems in Children. Journal of the American Academy of Child \& Adolescent Psychiatry, 56, 30-3937 https://doi.org/10.1016/j.jaac.2016.10.007 (2017).

15. Tuovinen, S. et al. Maternal antenatal stress and mental and behavioral disorders in their children. J Affect Disord, 278, 57-65 https://doi.org/10.1016/j.jad.2020.09.063 (2020).

16. Rogers, C. E., Lenze, S. N. \& Luby, J. L. Late preterm birth, maternal depression, and risk of preschool psychiatric disorders. J Am Acad Child Adolesc Psychiatry, 52, 309-318 https://doi.org/10.1016/j.jaac.2012.12.005 (2013).

Page $9 / 11$ 
17. Girchenko, P. et al. Cohort Profile: Prediction and prevention of preeclampsia and intrauterine growth restriction (PREDO) study. International Journal of Epidemiology, 46 (g), 1380-1381 https://doi.org/10.1093/ije/dyw154 (2016).

18. Goldenberg, R. L., Culhane, J. F., lams, J. D. \& Romero, R. Epidemiology and causes of preterm birth. The Lancet, 371, 75-84 https://doi.org/10.1016/S0140-6736(08)60074-4 (2008).

19. Rantalainen, V. et al. Polygenic prediction of the risk of perinatal depressive symptoms. Depression and Anxiety, 37, 862-875 https://doi.org/10.1002/da.23066 (2020).

20. Wolke, D. et al. Selective drop-out in longitudinal studies and non-biased prediction of behaviour disorders. Br J Psychiatry, 195, 249-256 https://doi.org/10.1192/bjp.bp.108.053751 (2009).

21. Gissler, M. \& Kiuru, S. Perinatal statistics: births, deliveries and newborns 2018(2019).

22. Girchenko, P. et al. Cohort Profile: Prediction and prevention of preeclampsia and intrauterine growth restriction (PREDO) study. Int $J$ Epidemiol, 46 (g), 1380-1381 https://doi.org/10.1093/ije/dyw154 (2017).

23. Radloff, L. S., The, C. E. S. D. \& Scale A Self-Report Depression Scale for Research in the General Population. Appl. Psychol. Meas, 1, 385401 https://doi.org/10.1177/014662167700100306 (1977).

24. Pesonen, A. K. et al. Maternal Prenatal Positive Affect, Depressive and Anxiety Symptoms and Birth Outcomes: The PREDO Study. PloS one, 11, e0150058 https://doi.org/10.1371/journal.pone.0150058 (2016).

25. Sund, R. Quality of the Finnish Hospital Discharge Register: a systematic review. Scand J Public Health, 40, 505-515 https://doi.org/10.1177/1403494812456637 (2012).

26. Kongsted, A. \& Nielsen, A. M. Latent Class Analysis in health research. J Physiother, 63, 55-58 https://doi.org/10.1016/j.jphys.2016.05.018 (2017).

27. Hayes, A. F. \& Rockwood, N. J. Regression-based statistical mediation and moderation analysis in clinical research: Observations, recommendations, and implementation. Behav Res Ther, 98, 39-57 https://doi.org/10.1016/j.brat.2016.11.001 (2017).

\section{Figures}

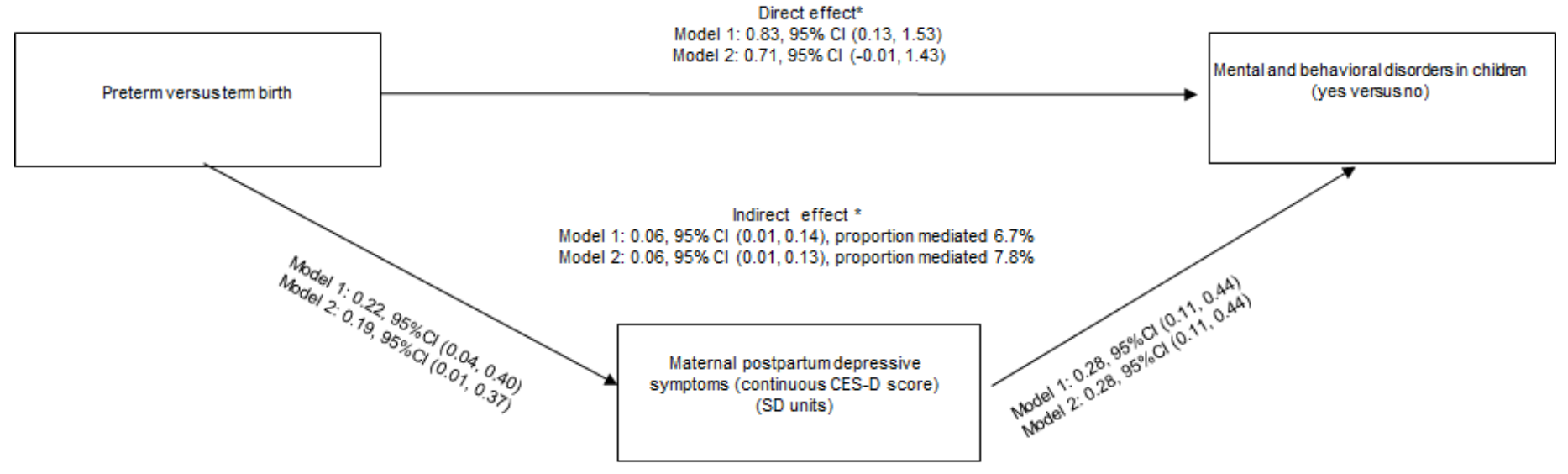

\section{Figure 1}

Mediation analysis showing that the effect of preterm birth on mental and behavioral disorders in children until age 8.4-12.8 years, is partially mediated via maternal postpartum depressive symptoms. Model 1 refers to unadjusted associations and Model 2 to associations adjusted for maternal age at delivery, education, smoking and/or alcohol use at any time during pregnancy, delivery mode and child sex and age at diagnosis. 

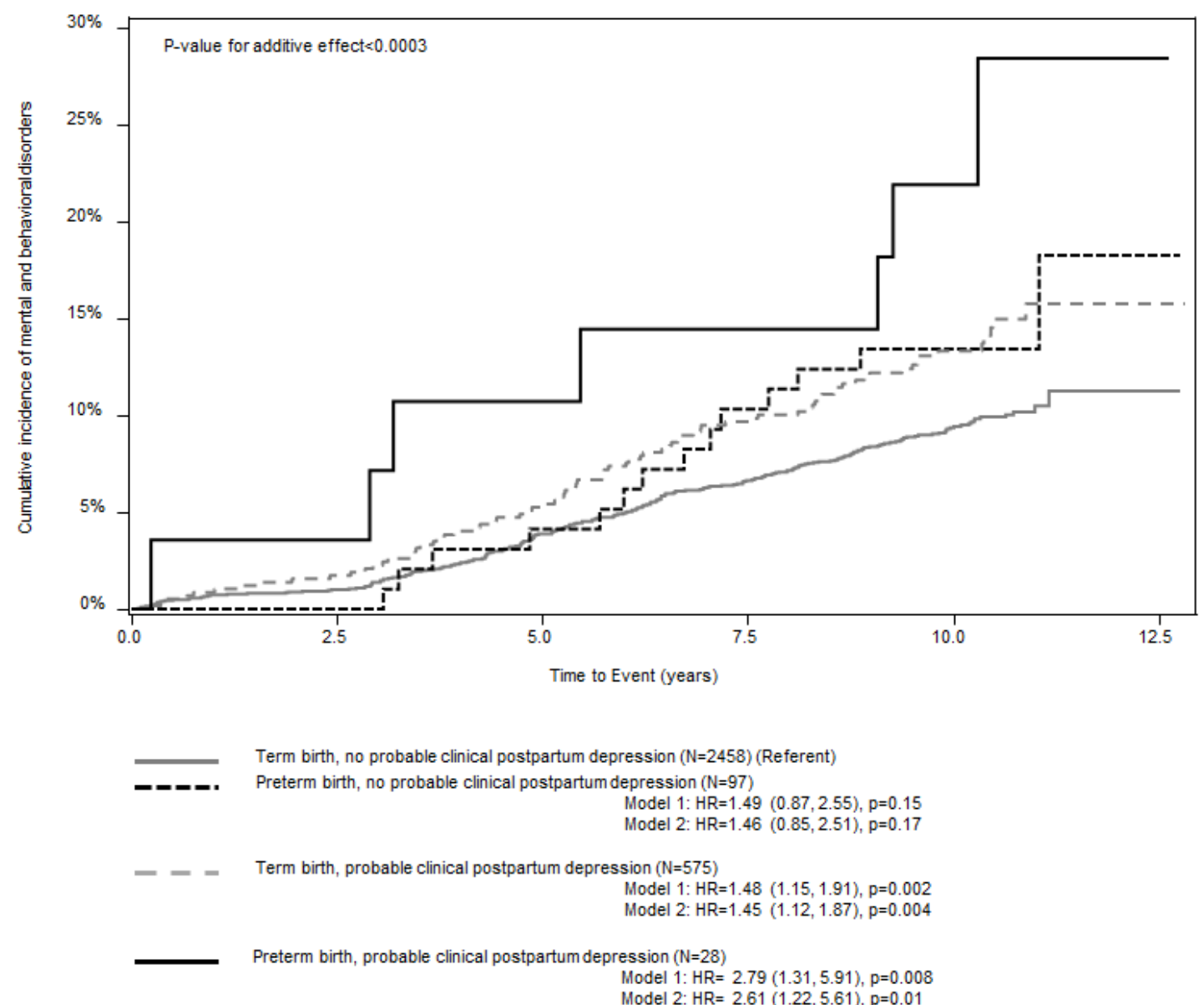

\section{Figure 2}

Additive effects of preterm birth and maternal postpartum depressive symptoms on mental and behavioral disorders in children. The lines represent cumulative incidence rates of mental and behavioral disorders until age 8.4-12.8 years in children born at term and whose mother reported no probable clinical postpartum depression (referent), (a) in children born preterm and whose mothers reported no probable clinical postpartum depression, (b) in children born at term and whose mother reported probable clinical postpartum depression, and (c) in children born preterm and whose mother reported probable clinical postpartum depression. HR refers to hazard ratio and $95 \% \mathrm{Cl}$ to $95 \% \mathrm{Confidence}$ Interval. Model 1 refers to unadjusted associations and Model 2 to associations adjusted for maternal age at delivery, education, smoking and/or alcohol use at any time during pregnancy, delivery mode and child sex. P-value for linearity refers to the additive effect of preterm birth and maternal postpartum depression ( $0=$ term, no postpartum depression, $1=$ preterm, no postpartum depression or term, postpartum depression, 2=preterm, postpartum depression).

\section{Supplementary Files}

This is a list of supplementary files associated with this preprint. Click to download.

- SupplementalTable1.docx

- SupplementalTable2.xIsx

- SupplementalTable3.docx

- SupplementalTable4.docx

- SupplementalTable5.docx 